\title{
Correlated imaging through atmospheric turbulence
}

\author{
Pengli Zhang, Wenlin Gong, Xia Shen and Shensheng Han* \\ Key Laboratory for Quantum Optics and Center for Cold Atom Physics, Shanghai Institute of Optics and Fine Mechanics, \\ Chinese Academy of Sciences, Shanghai 201800, China \\ *Corresponding author: sshan@mail.shcnc.ac.cn
}

Compiled October 26, 2018

\begin{abstract}
Correlated imaging through atmospheric turbulence is studied, and the analytical expressions describing turbulence effects on image resolution are derived. Compared with direct imaging, correlated imaging can reduce the influence of turbulence to a certain extent and reconstruct high-resolution images. The result is backed up by numerical simulations, in which turbulence-induced phase perturbations are simulated by random phase screens inserting propagation paths. (C) 2018 Optical Society of America
\end{abstract}

OCIS codes: 270.0270, 010.1330, 110.0115

As correlated imaging develops well in recent years [1-5], more attention has been focused on how to apply this technique to practical applications to overcome the limits in conventional optical systems. For an imaging system which must look through the atmosphere, turbulence-induced wavefront variations distort the point spread function (PSF) of the system from its ideal diffraction-limited shape, which leads to the the degradation of image resolution [6]. To mitigate turbulence effects, a number of methods, such as speckle imaging and adaptive optics techniques [6], have been proposed and applied in optical astronomy. Nonetheless, each of these techniques has its own set of performance limits, hardware and software requirements. New approaches to the problem of reducing these effects are still of much interest. Here we investigate the performance of correlated imaging through atmospheric turbulence and find that the influence of turbulence can be weakened by the second-order intensity correlation.

A schematic of correlated imaging through the atmosphere is depicted in Fig. 1. The beam splitter (BS) divides thermal light into two beams propagating through two distinct optical paths. One is test arm which includes an unknown object and a telescope setup consisting of a lens with focal length $f$ and a detector $D_{t}$. The object is located at a distance $d_{1}$ from the source as well as $d_{2}$ to the telescope setup. The other is the reference arm where another telescope setup consisting of a lens and a detector $D_{r}$ is placed at $d_{0}=d_{1}+d_{2}$ from the source. For remote sensing (i.e., $d_{1}, d_{2} \gg f$ ), the detector $D_{t}$ (or $D_{r}$ ) generally lies close to the back focal plane of the lens (i.e., $d_{3} \approx f$ ). The test arm is imbedded in the atmosphere, and turbulence-induced wavefront fluctuations in propagation paths $d_{1}$ and $d_{2}$ are represented by $\Psi_{1}$ and $\Psi_{2}$, respectively. While the reference arm is said to be a free-space propagation through the distance $d_{0}$ by assuming that there exists no turbulence. The assumption is based on the fact that the optical field in the reference arm is totally predictable if the field distribution of the source is well known $[1,2]$.

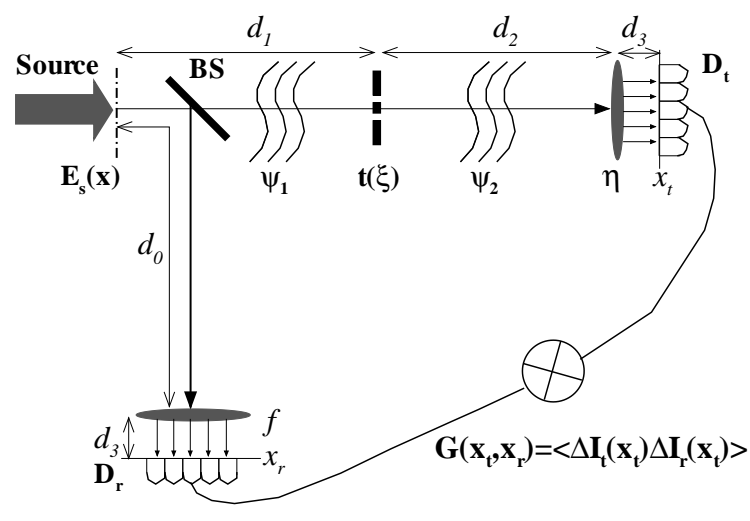

Fig. 1. Schematic of correlated imaging through atmospheric turbulence.

In the test arm, the field $E_{t}\left(x_{t}\right)$ in the detector $D_{t}$ can be given by

$$
E_{t}\left(x_{t}\right)=\iint d x d \xi E_{s}(x) h_{1}(\xi, x) t(\xi) h_{2}\left(x_{t}, \xi\right),
$$

where $E_{s}(x)$ corresponds to the source field, and $t(\xi)$ denotes the transmission function of the object. $h_{1}(\xi, x)$, $h_{2}\left(x_{t}, \xi\right)$ are the impulse response functions from the source to the object and from the object to the detector $D_{t}$, respectively.

Furthermore, according to the extended HuygensFresnel integral [7], $h_{1}(\xi, x)$ and $h_{2}\left(x_{t}, \xi\right)$ have the forms

$$
\begin{aligned}
& h_{1}(\xi, x)=\frac{1}{\sqrt{j \lambda d_{1}}} e^{\frac{j k}{2 d_{1}}(x-\xi)^{2}+\Psi_{1}(x, \xi)}, \\
& h_{2}\left(x_{t}, \xi\right)=\frac{1}{j \lambda \sqrt{d_{2} d_{3}}} \int d \eta e^{-\frac{j k}{d_{2}}\left(\xi-x_{t} / M\right) \eta+\Psi_{2}(\xi, \eta)},
\end{aligned}
$$

where $k=2 \pi / \lambda$ is the wave number with $\lambda$ being the wavelength, and $M=-d_{3} / d_{2}$ is the magnification of the telescope setup. $\Psi_{1}(x, \xi)$ and $\Psi_{2}(\xi, \eta)$ account for the random parts (due to atmospheric turbulence) of the complex phases of the fields in the propagation paths $d_{1}$ and $d_{2}$, respectively. 
The field $E_{r}\left(x_{r}\right)$ in the detector $D_{r}$ is connected to the source field $E_{s}(x)$ by the Fresnel diffraction integral

$$
E_{r}\left(x_{r}\right)=\frac{1}{\sqrt{j \lambda d_{1}|M|}} \int d x E_{s}(x) e^{\frac{j k}{2 d_{1}}\left(x-x_{r} / M\right)^{2}} .
$$

It's worth pointing out that the apertures of the lenses are regarded as large enough, and the diffraction limit of the lenses has been neglected here.

Performing the intensity correlation measurement between the test arm and the reference arm, we get

$$
\begin{aligned}
G\left(x_{t}, x_{r}\right)= & \left\langle I_{t}\left(x_{t}\right) I_{r}\left(x_{r}\right)\right\rangle-\left\langle I_{t}\left(x_{t}\right)\right\rangle\left\langle I_{r}\left(x_{r}\right)\right\rangle \\
= & c_{0} \int d x d x^{\prime} d x^{\prime \prime} d x^{\prime \prime \prime} d \xi d \xi^{\prime}\left\langle E_{s}(x) E_{s}^{*}\left(x^{\prime \prime \prime}\right)\right\rangle \\
& \times\left\langle E_{s}^{*}\left(x^{\prime}\right) E_{s}\left(x^{\prime \prime}\right)\right\rangle\left\langle h_{1}(\xi, x) h_{1}^{*}\left(\xi^{\prime}, x^{\prime}\right)\right\rangle \\
& \times\left\langle h_{2}\left(x_{t}, \xi\right) h_{2}^{*}\left(x_{t}, \xi^{\prime}\right)\right\rangle t(\xi) t^{*}\left(\xi^{\prime}\right) \\
& \times e^{\frac{j k}{2 d_{1}}\left[\left(x^{\prime \prime}-x_{r} / M\right)^{2}-\left(x^{\prime \prime \prime}-x_{r} / M\right)^{2}\right]}
\end{aligned}
$$

where $c_{0} \quad$ is a constant $\left(\lambda^{3} d_{1} d_{2} d_{3}|M|\right)^{-1}$, and $I_{t}\left(x_{t}\right), \quad I_{r}\left(x_{r}\right)$ represent the intensity distributions in $D_{t}$ and $D_{r}$, respectively. Here, we have supposed that the thermal field, and the two turbulent regions are statistically independent of each other.

If the source is fully spatially incoherent and its intensity distribution is of the Gaussian type, the first-order correlation function of the source has the form

$$
\left\langle E_{s}(x) E_{s}^{*}\left(x^{\prime}\right)\right\rangle=I_{0} e^{-\frac{x^{2}+x^{\prime 2}}{r_{e}^{2}}} \delta\left(x-x^{\prime}\right),
$$

where $I_{0}$ denotes the mean intensity at the center of the source, and $r_{e}$ is the $1 / e^{2}$ intensity radius. With the help of Eqs. (2a), (2b), and (5), Eq. (4) can be rewritten as

$$
\begin{aligned}
G\left(x_{t}, x_{r}\right)= & I_{0}^{2} \int d x d x^{\prime} d \eta d \eta^{\prime} d \xi d \xi^{\prime} t(\xi) t^{*}\left(\xi^{\prime}\right) \\
& \times e^{-\frac{2\left(x^{2}+x^{\prime 2}\right)}{r_{e}^{2}}} e^{\frac{j k}{2 d_{1}}\left[\left(x^{\prime}-x_{r} / M\right)^{2}-\left(x-x_{r} / M\right)^{2}\right]} \\
& \times e^{\frac{j k}{2 d_{1}}\left[(x-\xi)^{2}-\left(x^{\prime}-\xi^{\prime}\right)^{2}\right]}\left\langle e^{\Psi_{1}(x, \xi)+\Psi_{1}^{*}\left(x^{\prime}, \xi^{\prime}\right)}\right\rangle \\
& \times e^{\frac{j k}{d_{2}}\left[\left(\xi-x_{t} / M\right) \eta-\left(\xi^{\prime}-x_{t} / M\right) \eta^{\prime}\right]} \\
& \times\left\langle e^{\left.\Psi_{2}(\xi, \eta)+\Psi_{2}^{*}\left(\xi^{\prime}, \eta^{\prime}\right)\right\rangle .}\right.
\end{aligned}
$$

The ensemble average of phase variations arising from turbulence can be approximated by [7]

$$
\begin{aligned}
& \left\langle e^{\Psi_{i}(x, \xi)+\Psi_{i}^{*}\left(x^{\prime}, \xi^{\prime}\right)}\right\rangle \\
& \cong e^{-\frac{1}{\rho_{i}^{2}}\left[\left(x-x^{\prime}\right)^{2}+\left(x-x^{\prime}\right)\left(\xi-\xi^{\prime}\right)+\left(\xi-\xi^{\prime}\right)^{2}\right]},
\end{aligned}
$$

where $\rho_{i}=\left(0.545 C_{n}^{2(i)} k^{2} d_{i}\right)^{-3 / 5}(i=1,2)$ is the coherence length of a spherical wave propagating in the turbulent medium and $C_{n}^{2(i)}$ corresponds to the refractiveindex structure constants describing the strength of atmospheric turbulence in the propagation path $d_{i}$. It's worth emphasizing that we have adopted a quadratic approximation of the Rytov's phase structure function in Eq. (7) to obtain the analytical formula, and this approximation has been used widely in literatures $[4,7]$.
Substituting Eq. (7) to Eq. (6) and integrating over $\eta, \eta^{\prime}, x, x^{\prime}$, we have

$$
\begin{aligned}
G\left(x_{t}, x_{r}\right)= & \frac{\sqrt{\pi} I_{0}^{2} c_{0}}{\sqrt{\alpha \beta_{2}\left(\alpha+2 \beta_{1}\right)}} \int d \xi|t(\xi)|^{2} \\
& \times e^{-\frac{2 A^{2}}{\alpha+2 \beta_{1}}\left(\xi-x_{r} / M\right)^{2}} e^{-\frac{B^{2}}{\beta_{2}}\left(\xi-x_{t} / M\right)^{2}},
\end{aligned}
$$

where $A=k / 2 d_{1}, B=k / 2 d_{2}, \alpha=r_{e}^{-2} / 2, \beta_{i}=\rho_{i}^{-2}$.

By making $x_{r}=x_{t}$ in Eq. (8), we carry out a special point-to-point intensity correlation [8] and obtain the PSF of the correlated imaging system

$$
h_{g}\left(x_{r}, \xi\right)=e^{-\frac{2 A^{2}}{\alpha+2 \beta_{1}}\left(\xi-x_{r} / M\right)^{2}} e^{-\frac{B^{2}}{\beta_{2}}\left(\xi-x_{r} / M\right)^{2}} .
$$

For the sake of comparison, we also present the intensity distribution in $D_{t}$,

$$
I_{t}\left(x_{t}\right)=\frac{\sqrt{\pi} I_{0} c_{0}}{\sqrt{\alpha \beta_{2}}} \int d \xi|t(\xi)|^{2} e^{-\frac{B^{2}}{\beta_{2}}\left(\xi-x_{t} / M\right)^{2}},
$$

and the PSF of the test arm

$$
h_{t}\left(x_{t}, \xi\right)=e^{-\frac{B^{2}}{\beta_{2}}\left(\xi-x_{t} / M\right)^{2}} .
$$

From Eqs. (9) and (11), we can see that the full widths at half maximum (FWHM) of $h_{g}$ and $h_{t}$ both broaden with the increase of $\beta_{i}$ (apart from the influence of the size of the source), which indicates that the resolution, whether for correlated imaging or direct imaging, is degraded by atmospheric turbulence. Additionally, and most importantly, $h_{g}$ has a narrower FWHM compared to $h_{t}$, which means that correlated imaging is helpful to reduce turbulent effects and achieve high-resolution images.

In simulations, we consider correlated imaging through horizontal paths in the atmosphere, and thus $C_{n}^{2}$ can be regarded as constant in the whole turbulent regions. The numerical model of light propagation in turbulence has been developed well $[9,10]$. The spatial power spectral density of the index of refraction fluctuations can be described by the Von Karman spectrum [9],

$\Phi_{n}(K, z)=0.033 C_{n}^{2}(z)\left(K^{2}+L_{0}^{-2}\right)^{-11 / 6} e^{-\left(K l_{0} / 2 \pi\right)^{2}}$,

where $K^{2}=K_{x}^{2}+K_{y}^{2}+K_{y}^{2}, z$ is the propagation distance from the source, $L_{0}$ and $l_{0}$ represent the outer scale and inner scale of the turbulence, respectively. By using the spectrum in Eq. (12) to filter a complex Gaussian pseudorandom field and inverse transforming the result, one obtains a two-dimensional phase screen which has the same statistics as the turbulence-induced phase variations [9]. For long atmospheric paths, the multiple phasescreen model [10] has been used in simulations. The turbulent region with the propagation length $d_{i}$ is broken into a number of layers with a thickness $\Delta z$. Phase fluctuations in each layer are represented by a phase screen inserting in the middle of the layer. The effect of field propagation through these continuous layers can be calculated separately and then combined to characterize 


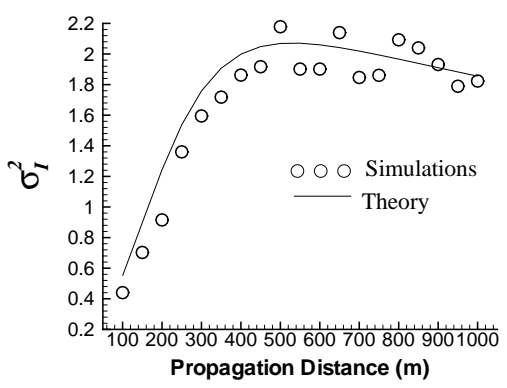

Fig. 2. Simulated ( open circles) and theoretical (solid line) on-axis irradiance variance versus the propagation distance. The outer scale and inner scale of turbulence are $L_{0}=3 \mathrm{~m}$ and $l_{0}=1 \mathrm{~cm}$, respectively.

propagation through the entire turbulent region, provided the index of refraction fluctuations for each layer are statistically independent [6].

First of all, to verify the computer programm, we investigate the behavior of a Gaussian beam (waist radius $w_{0}=7 \mathrm{~cm}$ and wavelength $\lambda=2 \mu \mathrm{m}$ ) traveling through the atmosphere with a strong turbulence level $\left(C_{n}^{2}=10^{-12} \mathrm{~m}^{-2 / 3}\right)$. The thickness of each layer is $\Delta z=50 \mathrm{~m}$. The on-axis normalized intensity variance, defined as $\sigma_{I}^{2}=\left\langle I^{2}\right\rangle /\langle I\rangle^{2}-1[9]$, is plotted as a function of the propagation distance in Fig. 2. The good coincidence between the simulated data (open circles) and the theoretical result (solid line) predicted by [11] proves the validity of the programm.

After the validation, we apply the programm to simulate the correlated imaging system shown in Fig. 1. The thermal source $\left(\lambda=0.532 \mu \mathrm{m}\right.$ and diameter $D=2 r_{e}=5$ $\mathrm{cm}$ ) was described by a grid of $512 \times 512$ with a sample spacing $\Delta x=\Delta y=5 \mathrm{~mm}$. The distances were set as $d_{1}=d_{2}=10 \mathrm{~km}$ and the focal length $f=1 \mathrm{~m}$. The turbulence regions in the paths $d_{i}(i=1,2)$ were divided into 20 layers with a thickness $\Delta z=500 \mathrm{~m}$, respectively. The turbulent parameters were assumed constant at the outer scale $L_{0}=100 \mathrm{~m}$ and the inner scale $l_{0}=5$ $\mathrm{mm}$. By averaging over $10^{4}$ samples, simulated results [see Fig. (3)] clearly show the image resolution decrease with the increase of the turbulent strength, which accords with the analytical calculation from Eq. (8).

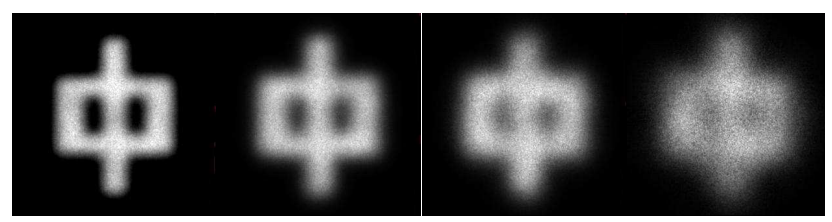

Fig. 3. The reconstructed images (from left to right) via the correlation in the atmosphere with turbulent levels $C_{n}^{2}=10^{-16} \mathrm{~m}^{-2 / 3}, 2.5 \times 10^{-16} \mathrm{~m}^{-2 / 3}, 5 \times 10^{-16} \mathrm{~m}^{-2 / 3}$, and $10^{-15} \mathrm{~m}^{-2 / 3}$, respectively.

To compare direct imaging and correlated imaging, a simple double slit (slit width $10 \mathrm{~cm}$ and center-to-center

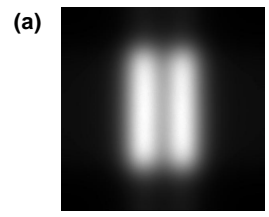

(b)
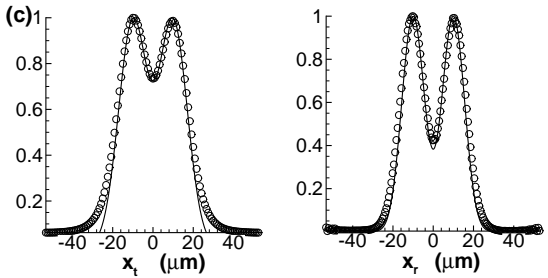

Fig. 4. The acquired images of the double slit in the atmosphere with turbulent level $C_{n}^{2}=10^{-15} \mathrm{~m}^{-2 / 3}$. (a) was obtained by the test arm, and (b) was extracted from the correlation. The normalized horizontal sections of the images are plotted in (c), where open circles correspond to the simulated data and solid lines show the theoretical predictions from Eqs. (8) and (10), respectively.

separation $20 \mathrm{~cm}$ ) was used. After statistics over $10^{4}$ samples, we obtained a blurred image detected by the test arm directly [see Fig. 4(a)] and a clear image reconstructed through the correlation [see Fig. 4(b)]. This confirms the analytical result that ghost imaging could reduce turbulent effects and improve resolution.

In summary, by taking advantage of the extended Huygens-Fresnel integral, we have presented the theoretical expressions that describes how atmospheric turbulence corrupts the image resolution. Meanwhile, the analytical calculations and the numerical simulations have demonstrated that correlated imaging can provide imaging performance superior to direct imaging through the atmosphere. As an unique image-formed method, correlated imaging can be effectively combined with conventional phase compensating techniques (e.g., adaptive optics) to further eliminate turbulent effects.

This work is supported by the Hi-Tech Research and development Programm of China (Grant No. 2006AA12Z115), Shanghai Fundamental Research Project (Grant No. 09JC1415000), and the National Natural Science Foundation of China (Grant No. 6087709).

\section{References}

1. Y. Bromberg, O. Katz, and Y. Silberberg, "Ghost imaging with a single detector," Phys. Rev. A 79, 053840 (2009).

2. J. H. Shapiro, "Computational ghost imaging," Phys. Rev. A 78, 061802(R) (2008).

3. Kam Wai Clifford Chan, M. N. O' Sullivan, and R. W. Boyd, "High-order thermal ghost imaging," Opt. Lett. 34, 3343 (2009).

4. Jing Cheng, "Ghost imaging through turbulent atmosphere," Opt. Express 17, 7916-7921 (2009).

5. W. Gong, P. Zhang, X. Shen and S. Han, "Imaging in scattering media via the second-order correlation of light 
field," arXiv.Quant-ph/0908.0185v1 (2009).

6. M. C. Roggemann, B. Welsh, Imaging through turbulence (CRC, USA, 1996).

7. J. C. Ricklin, and F. M. Davidson, "Atmospheric turbulence effect on a partially coherent Gaussian beam: implication for free-space laser communication," J. Opt. Soc. Am. 19, 1794-1802 (2002).

8. Pengli Zhang, Wenlin Gong, Xia Shen, Dajie Huang, and Shensheng Han, "Improving resolution by the secondorder correlation of light fields," Opt. Lett. 34, 12221224 (2009).

9. A. Belmonte, "Feasibility study for the simulation of beam propagation: consideration of coherent lidar performance," Appl. Opt. 39, 5426-5444 (2000).

10. D. L. Knepp, "multiple phase-screen calculation of the temporal behavior of stochastic waves," Proc. IEEE, 71, 722-736 (1983).

11. L. C. Andrews, M. A. Al-Habash, C. Y. Hopen, and R. L. Phillips, "Theory of optical scintillation: Gaussian-beam wave model," Waves in Random and Complex Media, 11. 271-291 (2001). 